\title{
COMPUTATIONAL INTELLIGENCE IN MULTI-SOURCE DATA AND INFORMATION FUSION ${ }^{1}$
}

\author{
Todor TAGAREV and Petya IVANOVA
}

\section{Introduction}

The rapid development of information technologies in the last decades of the twentieth century created opportunities to increase the effectiveness in practically every area of human activity. The use of ever more powerful computers contributed to a better understanding of the nature of many social, economic, physical and physiological phenomena. Somewhat surprising was the discovery that the increase of data collection and information processing power does not necessarily increase our ability to define cause-effect relationships and to predict the development of realworld processes. Computers helped us to discard certain paradigms and to reveal some characteristics of nonlinear behavior and complex interaction. Nevertheless, our abilities to analyze, model, and predict is still rather limited. A number of methods have been proposed that combine the power of computer processing with attempts to imitate biological processing and human intellect. A new framework recently emerged - computational intelligence (CI). The application of CI methods to the problem of fusing data and information from multiple sources has significant potential, not yet fully discovered.

Rapid developments in sensor and computing technologies allow to combine data and information from multiple sources. The advantages of combining outputs from a number of sources to increase performance has been long recognized in such diverse areas as political economy models, financial management, weather and climate prediction, estimation and prediction of physiological condition, diagnostics. The implication of effective fusion for the purposes of monitoring, situation assessment, early warning, and other security related issues is crucial. A small sample of examples includes: 
- The impact of the explosion of information technologies on the capabilities for political and military early warning, including the capabilities for detection and collection of threat indicators, the dissemination of potential warning signals and threat assessments, the interpretation of collected signals and patterns, the capabilities to understand, interpret and respond to collected warnings; ${ }^{2}$

- The impact of sensor and information processing technologies on organizations and systems for monitoring in peace-keeping, arms control and humanitarian operations; ${ }^{3}$

- Design and implementation of measures and systems for information security, information warfare and critical information protection; ${ }^{4}$

- Design of reliable and safe systems for individual landmine detection based on using ground penetrating radar with integration of multiple microwave-sensor technologies and development of multi-sensor data fusion, feature extraction and object classification methods and algorithms. ${ }^{5}$

Since the mid-80s, MultiSensor Data Fusion (MSDF) emerged as a powerful technology for handling large amounts of data and decision support. Data fusion is examined as the integration and application of both traditional disciplines and new areas of engineering to achieve the fusion of data. These areas include computer science, expert systems, communication and decision theory, epistemology, estimation theory, digital signal processing, fuzzy logic, and neural networks. Methods for representing and processing data (signals) are adapted from each of these disciplines to perform data fusion.

Rapid developments in the field of information technology created opportunities for qualitative increase in data storage, processing power, and presentation. On that basis, the MSDF processes, including collection of data from multiple sensors, association, aggregation, and merging of data to increase the understanding of past and current situations, provided new opportunities. Ultimately, the output from a data fusion system is aimed at supporting a human decision process. The usefulness of a fusion system is measured by the extent to which the system supports the intended decision process.

In the process of decision making most people can not process rationally large quantities of data rapidly and accurately. But, as a rule, people deal well with situations, characterized by incomplete, imprecise, and uncertain information. Therefore, to adequately support the decision process, we need 'technologies' that, while processing increasing amounts of data, exploit the human tolerance for imprecision, uncertainty, and partial truth. Such a technology is computational intelligence. 
In the current paper, we examine potential applications of computational intelligence methodologies to support decision making by fusing data and information from multiple sources. In section II we briefly describe the well known data fusion and decision support system architecture for command and control, based on the SHOR decision making model. Section III presents this model in the framework of a new view on information space. Section IV outlines basic ideas of computational intelligence, and section $\mathrm{V}$ - its application in developing systems and algorithms for data and information fusion and decision support. As an example, in section VI we describe the application of CI methodologies in two ongoing projects. We conclude by emphasizing the potential for expanding traditionally military technologies and their contribution to increasing stability and security.

\section{MSDF and Decision Support in Military Applications}

The decision making model proposed by Wohl in studying command and control is depicted on Figure $1 .^{6}$ The SHOR model comprises four dynamically interacting elements:

- Stimulus - The initiation of the decision making process to provide information on the current situation and the associated uncertainties;

- Hypotheses - A set of perception alternatives explaining the real-world situation;

- Options - Response alternatives made available to the decision maker;

- Response - The selected action to be taken.

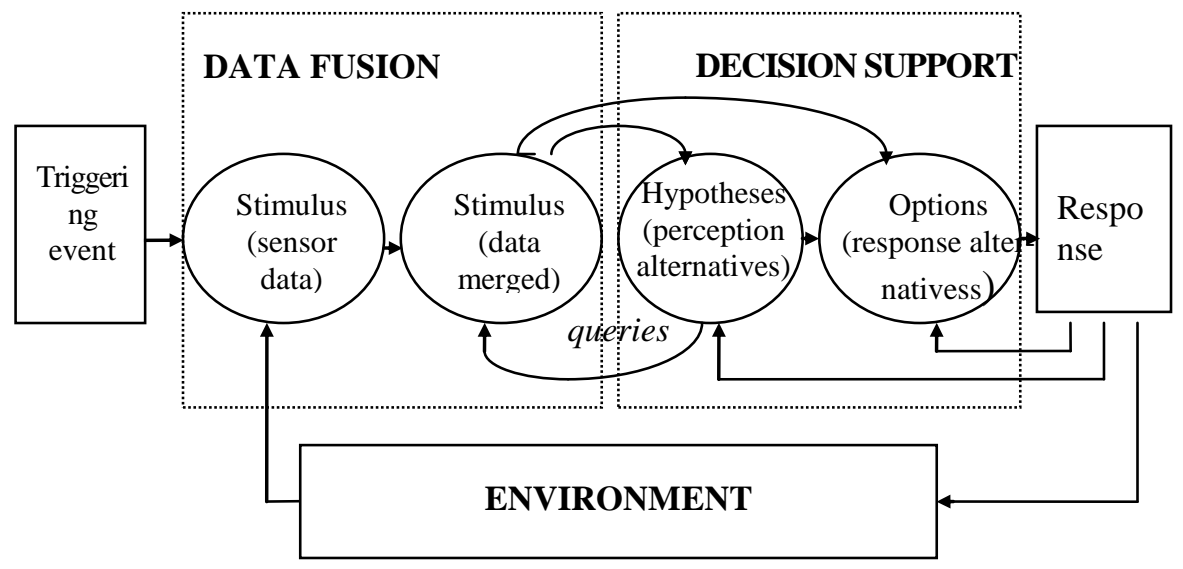

Figure 1: Data fusion and decision support in the SHOR model 
Waltz and Buede divide the 'information' part of the model into two distinct subsystems for data fusion and decision support. ${ }^{7}$ Data fusion collects information from various sensors and sources in order to develop the best possible perception of the situation. The situation is described by friendly and enemy orders of battle, locations and movements of weapons and equipment, events, and intelligence as it relates to past, present, and predicted behavior of the enemy. In the fusion process the authors include collection, association, aggregation, and merging of data to create and display current and past situations. The decision support function creates and evaluates alternative estimates of the real situation and the responses available to the commander. Both functions are performed interactively, and the results of the military response are included in the model through a feedback loop.

\section{MSDF vs Multisource Information Fusion}

In 1986, Prof. Arapov published an overview of the developing information technologies and their societal impact. ${ }^{8}$ For that purpose he proposed a model of the information space. Twelve years later a modified version of his model was announced, called Stratified Information Space Model. ${ }^{9}$ Both models allow to study IT developments and influence in three strata: data and signals, knowledge, and culture. Accordingly, we propose a modification of the SHOR model, depicted in Fig. 2 .

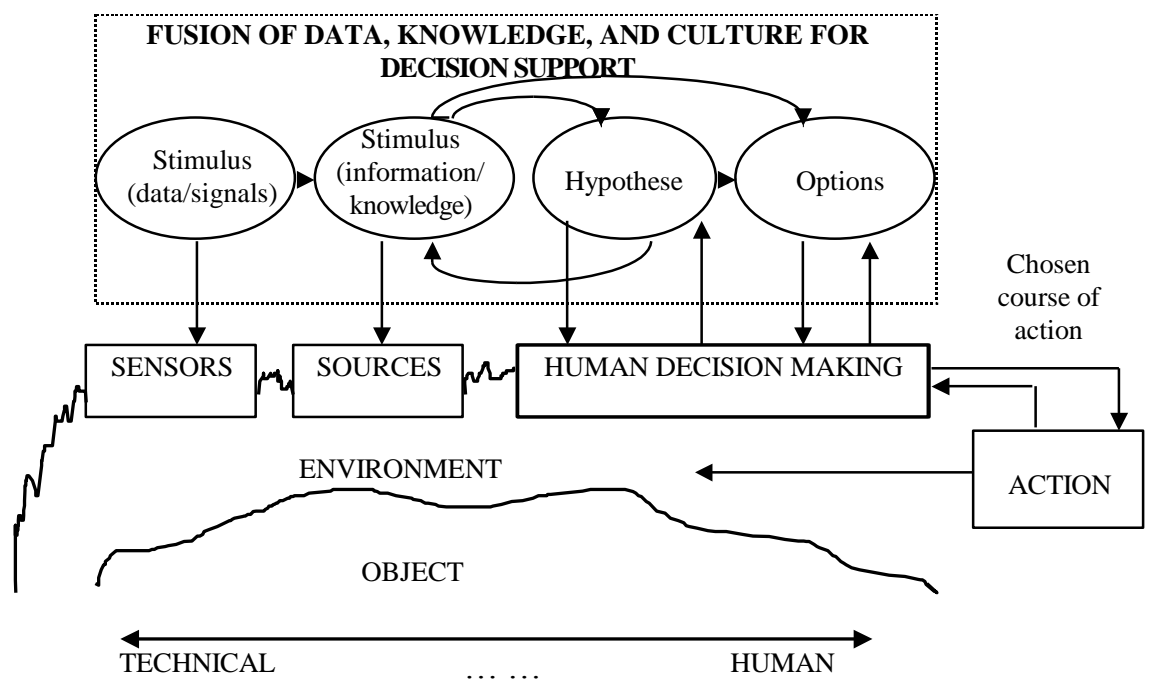

PROCESSING

Figure 2: MultiSource Information Fusion (MSIF) for decision support 
This modification allows for a better representation of the information strata DataKnowledge-Culture and gives a preference to the term source, examining sensors as just one type of sources. Respectively, the type of processing along the information strata changes from exclusively technically oriented to increasing human participation.

The MSIF model represents the intersection between the MSDF framework ${ }^{10}$ and the information space paradigm. ${ }^{11}$ There are two main differences between the classic SHOR model and the MSIF model:

1) We examine the term information more broadly to include not only technologically acquired data from the object under study and its environment, but also:

- inputs based on expert analysis, i.e., intelligence forecasts;

- criteria-type inputs for the decision making process based on value systems, doctrine, education and training, etc.

2) Instead of response we propose the use of term action. The difference is not just linguistic but in the essence. The term response assumes reactive attitude while, as in the cases under examination in section VI, we aim at taking preventive measures.

The outlined differences are not crucial. Nevertheless, they have methodological implications and, in our opinion, the MSIF model will help to expand the application of proven MSDF techniques to diverse problem areas. And although in many cases there is an overlap between various strata of the information space and variety of combinations between roles of technology and experts, this model allows for a unified framework clearly separating functions in the system from methods dealing with partial, uncertain, and imprecise information.

At this juncture, several approaches to MSDF were proposed in order to deal with incompleteness and ambiguousness both in the available data and in the preferences of decision makers. In terms of methodology, a minimal representative sample includes contributions from fuzzy set theory, neural networks, probabilistic reasoning, and multiple-criteria decision making under uncertainty:

- Waltz and Buede use the term soft decision data - representation of uncertainty using probabilities, possibilities, or fuzzy rules - as opposed to hard decisions (declarations). Hard decisions are reported as single statements, and soft decisions are provided as multiple hypotheses, each with its own representation of the uncertainty associated with the hypothesis. ${ }^{12}$ When fuzzy rules are used, 
uncertainty is reflected both in the fuzzy character of the if-then rules and the fuzzy presentation of the input information through membership functions.

- Studying the approaches to identity declaration in MSDF, Hall examines the potential for application of adaptive neural networks (NNs) for pattern recognition. ${ }^{13}$ On the next level of the MSDF system - decision level identity fusion - he examines the use of classical inference, Bayesian inference, Dempster-Shafer method, generalized evidence processing theory and heuristic methods for association/fusion of identity declarations from different sources.

- Rao studies the capacity of neural networks to fuse data and information when the error densities of the separate sources are unknown. ${ }^{14}$ Many of the existing information integration techniques are based on maximum a posteriori probabilities of hypotheses under a suitable probabilistic model. However, in situations where the probability densities are unknown (or difficult to estimate) such methods are ineffective. Therefore, as opposing to early methods (many of which required even independence of the errors of the sources), he envisions NN schemes that extract/infer fusion rules on the basis of empirical data and employ suitable training algorithms. Furthermore, Rao proves that for a certain class of continuous functions a feedforward neural network infers fusion rules that provide empirical risk minimization.

- Decision making involves choosing some course of action among various alternatives. In almost all decision making problems, there are several criteria for judging possible alternatives. The main concern of the decision maker is to fulfill his or her conflicting goals while satisfying the constraints of the system. Milakooti and Zhou formulate the multiple criteria decision making problem and use an adaptive $\mathrm{NN}$ to rank the set of discrete alternatives where each alternative is associated with a set of conflicting and noncommensurate criteria. ${ }^{15}$ Examining decision making problems under certainty, they consider discrete sets of alternatives with the assumption that there exists a multiple attribute utility function (MAUF) that can represent the preferences of the decision maker. They demonstrate that adaptive NNs can represent a more general and flexible MAUF than other generally used types of MAUFs. Adaptive NNs for representing various MAUFs enable the decision maker to rank alternatives and choose the most desirable ones. The authors show that the NN approach to solve multiple criteria problems is versatile yet robust approach to quantification and representation of the preferences of the decision maker: First, it does not assume any particular structure or property of MAUF; secondly, the NN method generates a completely assessed function; and, third, it can adjust and improve its representation as more information from the decision maker becomes available. 
The cited works provide but a glimpse at the power of fuzzy logic and neural networks to deal with complex processes in the lack of certainty and precision and to learn by example. Even more promising is their combined implementation, integrated with powerful optimization techniques in a probabilistic framework.

\section{Computational Intelligence and Soft Computing Methodologies}

Recently, the term 'computational intelligence' is gaining influence in analysis, modeling and control of complex processes. It describes a concept for synergistic implementation of information processing methods in parallel with levels of human information processing. Figure 3 depicts this parallel. On the left side is the 'biological' processing according to the idea of the "triune brain". ${ }^{16}$ It envisions a cortex organized in three layers responsible respectively for instinctual behavior, motivational and emotional influences, and rational influences on decision making. The parallel with computational intelligence is presented on the right side of Fig. 3. The three respective layers involve implementation of quantitative statistical methods, soft-computing, and rule-based approaches of the symbol-processing kind. ${ }^{17}$

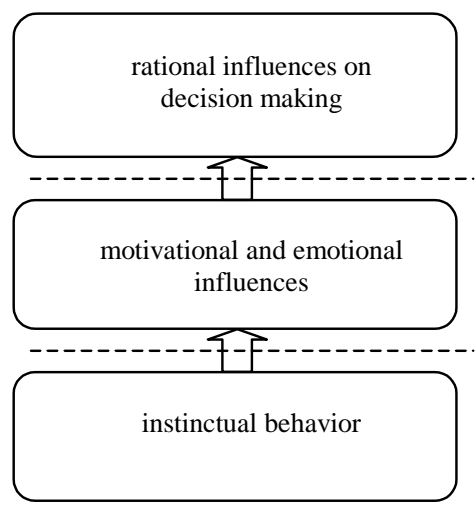

Human information processing

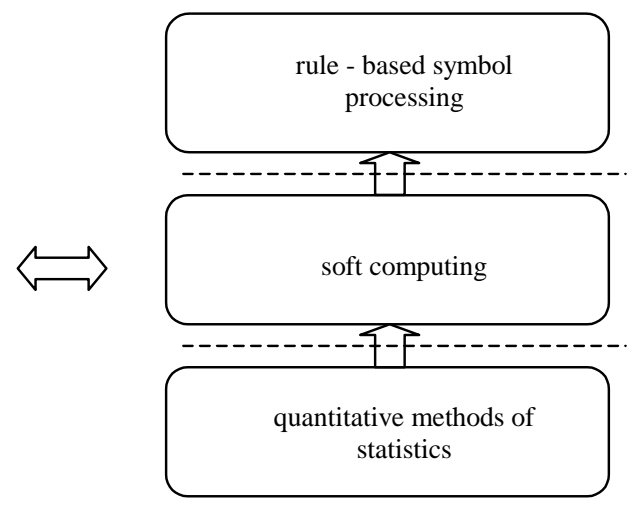

Computational intelligence

Figure 3: Parallel between human and computational intelligence

Guiding principle of the implementation of soft computing is the exploitation of the tolerance for imprecision, uncertainty and partial truth to achieve tractability, robustness and low solution cost. In 1994, Prof. Zadeh considered as main components of soft computing fuzzy logic, neural network theory, and probabilistic reasoning, the latter including parts of evolutionary computation, parts of learning theory, belief networks, and chaos theory. ${ }^{18}$ Of these, the first principal component is primarily concerned with imprecision of data and information, the second - with 
learning, and the third - with uncertainty. Soft computing is not a single methodology but rather a consortium of methodologies. At this juncture, fuzzy logic, neural networks, probabilistic reasoning and genetic algorithms are considered as principal constituents of soft computing. In many applications it is advantageous to exploit the synergism of these methods by using them in combination rather than alone. Examples of combined use include neuro-fuzzy, neuro-genetic, neuro-probabilistic, fuzzy-probabilistic, genetic-fuzzy and neuro-fuzzy-genetic systems. ${ }^{19}$

\section{Soft Computing in MSIF and Decision Support}

The focus of the Berkeley group led by Prof. Zadeh is on the development of techniques for combined implementation of fuzzy, neural, genetic, and probabilistic methods in the design of autonomous systems. ${ }^{20}$ We study the application of soft computing methodologies in MSIF for decision support. Accordingly, we transform the SHOR model (Fig. 2) to a MSIF model (Fig. 4) that presents functional problems in $\mathrm{MSDF}^{21}$ as problems of information fusion in the face of imprecision, uncertainty, high complexity, and change.

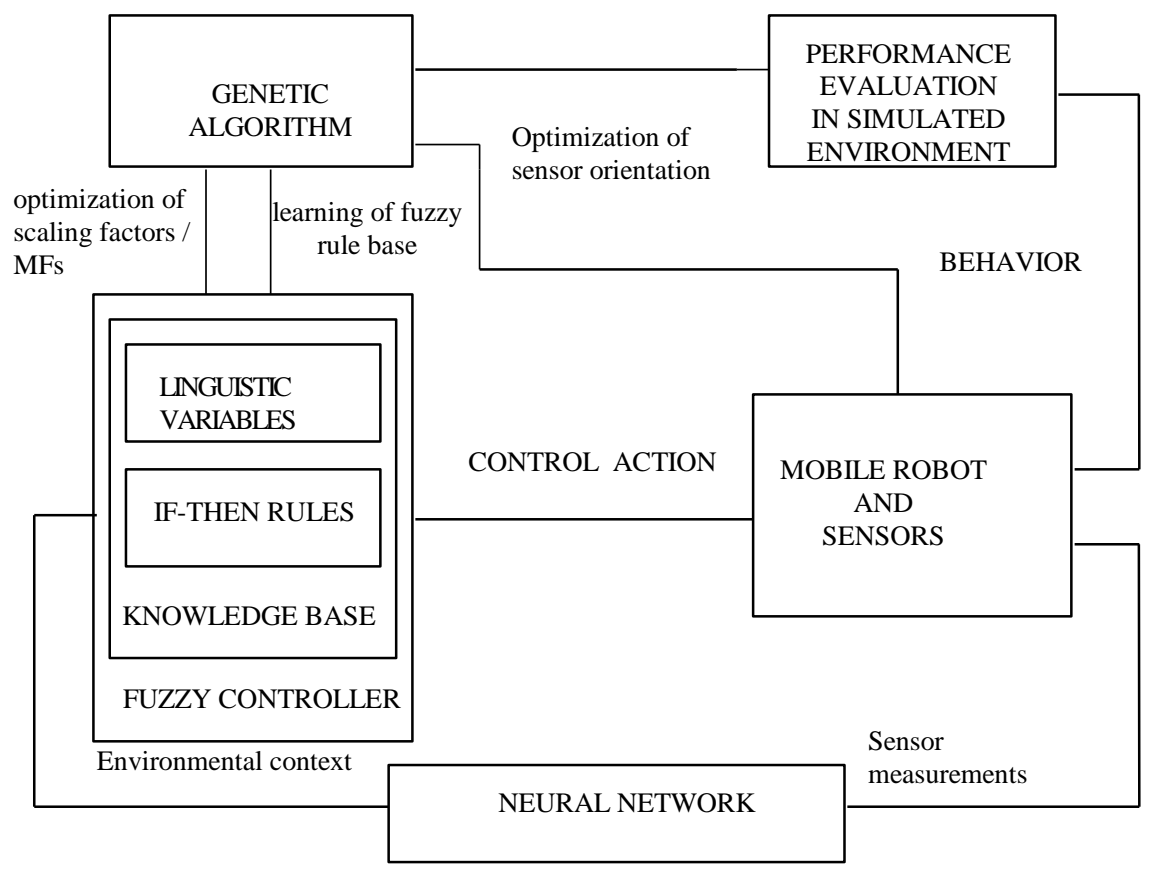

Figure 4: MSIF in the Information-decision-Action (IDA) loop 
In this dynamical framework, principal tasks in fusing information from multiple sources are:

- Classification - placing the current situation in a class of typical behavior. This goal is achieved when we have a priori (usually expert) knowledge of the types of behavior allowing to implement supervised learning methods. If such knowledge is not available, solving the task involves formation of classes via unsupervised learning;

- Modeling - finding a description that accurately captures features of the longterm dynamical behavior of the system;

- Characterization - determining fundamental properties of the system with little or no a priori knowledge;

- Forecasting - accurately predicting the short-term evolution of the system.

These tasks are overlapping but not necessarily identical. They serve as building blocks for event forecasting (Fig. 5), which we regard as the basis for decision support. Details are provided in the next section.

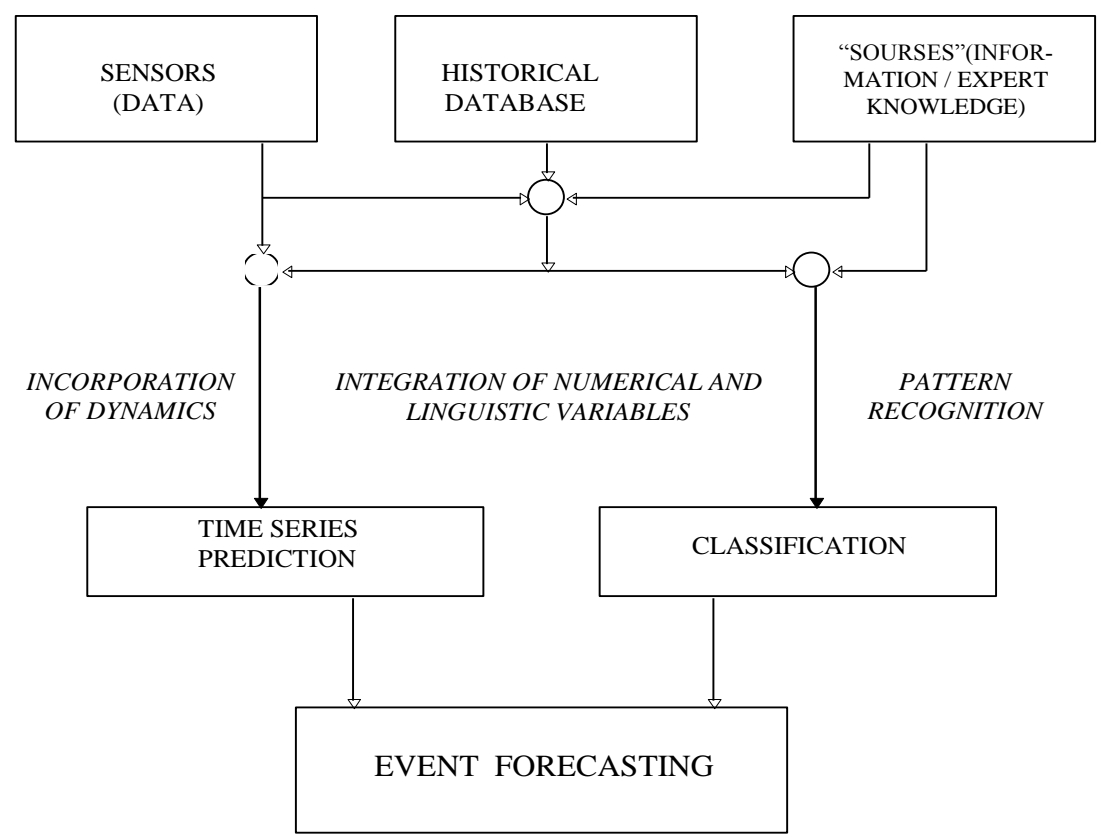

Figure 5: Functional tasks in event forecasting 


\section{Computational Intelligence for Early Warning}

Soft-computing methodologies are applied in two ongoing projects at the Space Research Institute of the Bulgarian Academy of Sciences.

\subsection{Classification of physiological condition for advance warning}

Multisource fusion of physiological information is a promising venue in the analysis and forecasting of biological and psychological processes. A R\&D group at the Space Research Institute designed the 32-channel system "NEUROLAB-B". The system, used on the "Mir" space station, records four channels of ECG, four channels of EMG, two channels of EOG, blood pressure, pulse wave, breath frequency, two channels body temperature, skin conductance level, dynamometric probe and somatosensor stimulation, and is working on a Holter system for 24-hour recording. ${ }^{22}$ Up to now, data is being processed by traditional statistical and signal processing techniques. However, recent research results convincingly ascertained the nonlinearity of many physiological processes and the complex interaction of variety of factors. ${ }^{23}$ On the basis of this multisource information the research group started the development of novel nonlinear methods and algorithms to forecast physiological condition of an operator working under external conditions.

\subsection{Early warning through classification of security situations}

Effective prevention of crisis or moderation of their impact requires advance warning. The application of traditional statistical and knowledge-based approaches to early warning did not prove sufficiently powerful for the purposes of early warning. That is not surprising if we account for the highly nonlinear character of triggering mechanisms and the levels of uncertainty caused both by the imprecise and partial information and by the inherently complex dynamics of security processes. ${ }^{24}$

In the quest for efficiency, the use of artificial NNs has been suggested. After transforming the expert knowledge into a representative set of indicators in a form suitable for $\mathrm{NN}$ processing, they are fed into an $\mathrm{NN}$ recognition scheme, trained on a historical data base. Thus, advance warning is issued when a particular security situation is recognized as a preconflict situation.

Both studies are aimed at providing advance warning. Although to a different degree, the resulting systems must support decisions in the face of poorly defined situations, with poorly measured variables, incomplete and inaccurate data, and incomplete theoretical understanding. We aim at a new quality both in the accuracy of prediction and in presenting information in the language used by decision makers through combined application of computational intelligence as follows. 


\subsection{Computational intelligence on the implementation stage}

To classify certain situation as a potential pre-crisis situation we apply a combination of fuzzy logic, NNs and probabilistic computing. Fuzzy representations of the input information reflect the symbolic nature of the expert opinion. The use of fuzzy sets allows to employ a mode of approximate reasoning, to describe knowledge by linguistic concepts, and to make decisions based on imprecise and incomplete information in a way similar to human beings. However, the sheer amount of information precludes the inference of a complete set of fuzzy rules. Therefore, we take advantage of the learning capabilities of NNs. The combination of a particular form of neural network, i.e. multilayer perceptron (MLP), with the transparent knowledge representation of fuzzy systems, produces a model with the ability to learn from real world observations and whose behavior can be described naturally as a series of linguistic humanly understandable rules.

Furthermore, instead of seeking a single optimal event forecasting model, we envision the use of several potential predictive models, which may lead to different inferences. Intuitively, ambiguity over the model should dilute information about predictions. A promising technique for properly accounting for this source of uncertainty, as well as for interpretation of the output of the classification scheme (type of expected crisis) is the Bayesian model averaging. Of considerable importance is the fact, that the treatment of the neuro-fuzzy system from a Bayesian perspective leads to practical procedures for estimating the confidence in the predictions.

Finally, it is possible that not a single configuration of inputs points to an upcoming crisis, but the way in which a configuration evolves in time. Hence, we need to build a warning system with inherent dynamic behavior. But MLPs are purely static and incapable of processing time information. Several approaches for incorporation of the dynamics in classification and forecasting schemes have been successfully applied:

- One way to extend MLPs to time processing is by creating a time window over the input configurations to serve as memory of the past. This leads to the so called time-delay NNs;

- Alternatively, recurrent NNs may be applied. The latter, however, are not guaranteed to be stable and they cannot be trained with standard backpropagation. These problems are avoided in a scheme known as partially recurrent network; ${ }^{25}$

- A third approach, devised by one of the authors ${ }^{26}$ involves design of nonlinear predictive models of the dynamics of some input variables and the use of the parameters of the model as potential features in the input space of the classification scheme. A similar approach, involving global dynamical models of 
the data, was used successfully to classify high noise signals, such as actual open ocean acoustic data. ${ }^{27}$

\subsection{Computational intelligence on the design stage}

One of the goals in design is to develop methods that account for prior knowledge of data and exploit such knowledge in reducing search and that, in the same time, are robust against uncertainty and missing data problems. Bayesian methods and decision analysis provide a basic foundational framework. This framework is successfully supplemented by contributions of chaos theory and genetic algorithms in the definition of an informative input space, the choice of the structure of the forecasting model, and the calibration of its parameters (the learning phase).

Design of input space. Chaos theory is applied to estimate the dimension of possible attractors in the situational dynamics. The estimated attractor dimension is used to initialize the number of input indicators of the classification scheme. Then, the genetic algorithm technique is used to precisely configure the indicator space so that it accounts for both dimensional and time (memory-type) factors. ${ }^{28}$ At this stage the dimensionality of the input data is reduced and deficiencies such as missing input values or incorrect target values are eliminated.

Structure of the forecasting scheme. Requirements for tractability (overcoming the curse of dimensionality) and model generalization (expanding the predictive power of the classification scheme to previously unseen situations) give significant advantage to parsimonious models. Furthermore, such models provide for a qualitative insight into the behavior of the system in the form of fuzzy rules. Therefore, we formulate the design as an optimization problem. The genetic algorithms are well suited for optimization in high-dimensional, nonlinear and noisy problems. They offer a means to systematically and efficiently explore the space of forecasting architectures. Also, they allow to optimize concurrently the NN topology, its parameters, as well as the parameters of the learning algorithm.

An alternative is provided by the Bayesian approach. Using only the training data, it allows different models to be compared in an objective and principled framework for dealing with the issues of model complexity. Also, estimations of the relative importance of different inputs can be automated. Choices can be made as to where in input space new data should be collected in order that it be most informative (such use is known as active learning).

Learning. Many algorithms exist for optimizing the values of the parameters in the network, in other words, for training the network. Successful solutions of various problems apply latest developments in machine intelligence allowing to mimic the ability of the human mind to effectively employ modes of reasoning that are 
approximate rather than exact, to learn from past experience, and to adapt to environmental changes. ${ }^{29}$ In most cases, we have expert-defined pairs "input configuration of indicators - class of situation." Then we apply algorithms for supervised learning, closely approximating available pairs (training examples). Examples for such algorithms in NN learning are back-propagation and simulated annealing.

When expert knowledge is lacking or insufficient, unsupervised learning techniques are applied for data clustering. Central data clustering, also called vector quantization, and pairwise data clustering are two classes of combinatorial optimization methods for data grouping which extract hidden structure from data. The main issues in algorithm development are to determine appropriate quality measures for the evaluation of clusters and to limit the complexity of the cluster set. Several models for data clustering exist, e.g., K-means clustering, selforganizing feature maps, the neural gas algorithm and complexity optimized vector quantization. Respective algorithms to estimate the cluster parameters have been derived in the maximum entropy framework which has been proven to be optimal for stochastic optimization. Clustering models for proximity data have also been introduced. Proximity data characterize items by their mutual relationship and not by coordinates in a vector space. ${ }^{30}$

Data issues. For most applications before training the classification/forecasting scheme it is necessary to transform the data into some new representation. Because of the very few assumptions in using computational intelligence methods, this problem is alleviated to some extent and less emphasis has to be placed on careful optimization of pre-processing than would be the case with simple linear techniques, for instance. Nevertheless, in most practical applications pre-processing of available data has a significant influence on the performance of the final system. Computational intelligence methods find successful applications in: ${ }^{31}$

- Input normalization;

- Input encoding;

- Dimensionality reduction: feature selection, ${ }^{32}$ feature extraction;

- Pre-filtering, removal of outliers;

- $\quad$ Dealing with missing data;

- Integration of domain specific knowledge.

Similarly, CI methods are used for post-processing to provide required output data. 


\section{Conclusion}

The application of computational intelligence in our studies extends the potential of 'traditional' MSDF. The discipline of multisensor data fusion appeared as a particular technology to support command and control. Thus, our extension of MSDF may be examined as a special type of convergence of soft 'military technologies' to non-military applications.

Of particular interest is the application of modern information technologies to increase international stability. Early warning through close international cooperation has a great potential to defuse crisis and conflict even before they appear. The monitoring of security situations for the purposes of early warning is such application calling for allsource data and information fusion, analysis, assessment and decision making support. ${ }^{33}$ Therefore, through development of architectures for multisource information fusion, the application of computational intelligence may contribute to increasing stability and security.

\section{Acknowledgment}

The work on this paper was partially supported by Bulgarian National Science Fund under grant I-621-96.

1 This article expands and further elaborates the ideas in a paper by the same authors, presented at the 1998 AFCEA Kiev Seminar: Todor Tagarev, Petya Ivanova and Anton Spasov, "Soft Computing Methodologies for MultiSource Information Fusion", in: The Information Technology Contribution to the Building of a Safe Regional Environment, ed. Vladimir Zaslavskii and Emmanuel Greindl, (Kiev: AFCEA, 1998), 31-44.

Max V. Metselaar, "Is more Necessarily Better? Advantages and Disadvantages of the Explosion of Information Technologies for Political and Military Preparedness," in 
Information Operations, ed. J.M.J. Bosch, H.A.M. Luiijf and A.R. Mollema (RMA Breda: NL ARMS - Netherlands Annual Review of Military Studies, 1999), 259-274.

Jurgen Altmann, Horst Fisher and Henny van der Graaf, eds., Sensors For Peace: Applications, Systems And Legal Requirements For Monitoring In Peace Operations (New York: United Nations Publication, 1998); Péricles Gasparini Alves, ed., Increasing Access to Information Technology for International Security. Forging Cooperation among Research Institutes in Europe, the United States and Canada (Geneva: United Nations Institute for Disarmament Research, 1998).

4 Willi Stein, "Information Warfare in the Context of Security-related Issues," in Information Operations, ed. J.M.J. Bosch, H.A.M. Luiijf and A.R. Mollema (RMA Breda: NL ARMS - Netherlands Annual Review of Military Studies, 1999), 181-198.

International project "Advanced Re-Locatable Multi-Sensor System for Buried Landmine Detection" coordinated by the International Research Center for Telecommunicationstransmission and Radar at the Technical University of Delft, The Netherlands. More information on the project is available in Internet at http://irctr.et.tudelft.nl/projects/tara/frames.htm. Some technical details are described in A.G. Yarovoy, B.Sai, G.Hermans, P.van Genderen, L.P.Ligthart, A.D.Schukin, and I.V.Kaploun, Ground Penetrating Impulse Radar for Detection of Small and ShallowBuried Objects, Proceedings of IGARSS 5 (Hamburg, Germany, June 28 - July 2, 1999), 2468-2470.

Edward L. Waltz, and Dennis M. Buede, Data Fusion and Decision Support for Command and Control, IEEE Transactions on Systems, Man, and Cybernetics SMC-16, 6 (November-December 1986), 865-879.

7 Waltz and Buede, "Data Fusion and Decision Support."

8 M.V. Arapov, "Society Informatization: Foreign Experience," Vestnik Academii Nauk USSR (1986), 62-76.

9 Tzvetan Semerdjiev, "The Impact of the MSDF Technologies on the Information Space Structure Reordering and Evolution," Information \& Security 1, 1 (Summer 1998), 75-90.

David Hall, Mathematical Techniques in Multisensor Data Fusion (Boston: Artech House, 1992).

Semerdjiev, "The Impact of the MSDF Technologies."

Waltz and Buede, "Data Fusion and Decision Support."

Hall, "Mathematical Techniques."

Nageswara S.V. Rao, "Fusion Methods for Multiple Sensor Systems with Unknown Error Densities," Journal of the Franklin Institute 331B, 5 (1994), 509-530; Nageswara S.V. Rao, "Multiple sensor fusion under unknown distributions," Journal of the Franklin Institute 336, 2 (1999), 285-299.

Behnam Malakooti and Ying Q. Zhou, Feedforward Artificial Neural Networks for Solving Discrete Multiple Criteria Decision Making Problems, Management Science 40, 11 (November 1994), 1542-1561.

16 The idea of the "triune brain" is proposed by Paul D. Mclean in The Triune Brain in Evolution: Role in Paleocerebral Functions, (N.Y: Plenum, 1990), 519-563. Initially, the parallel is drawn in Raju S. Bapi and Micheal J. Denham, "Computational Intelligence: a 
Synergistic Viewpoint," Computational Intelligence in Software Engineering 1,1 (March 1996).

Bapi and Denham, "Computational Intelligence."

Lotfi A. Zadeh, "Fuzzy Logic, Neural Networks, and Soft Computing," Communications of the ACM 37, 3 (March 1994), 77-84.

19 For introduction to the constituents of soft computing refer to Frank Hoffmann, "Soft Computing Techniques for the Design of Intelligent Systems," OAI Workshop on Fuzzy Logic (Ohio Aerospace Institute, 1997). Available on-line at http://www.cs.berkeley.edu/ fhoffman/oai97/oai97.html and the references therein. Hoffmann, "Soft Computing Techniques for the Design." Hall, "Mathematical Techniques."

Svetozar Simeonov, Stoyan Tanev and Plamen Trendafilov, "Neurolab-B' - A System for Psycho-physiological Studies on Board of the 'Mir' Station," Second Conference on Space, Aviation and Sea Medicine, (Varna, Bulgaria: October 1997).

See for example Holger Kantz, Juergen Kurths and Gottfried Mayer-Kress, Nonlinear Analysis of Physiological Data (Berlin: Springer Verlag, 1998).

For qualitative analysis and quantitative evidence the reader may refer to David S. Alberts and Thomas J. Czerwinski, eds., Complexity, Global Politics and National Security (Washington, DC: INSS, National Defense University, 1997); Saperstein, Alvin M., Dynamic Modeling of the Onset of War (Singapore: World Scientific, 1999); Todor D. Tagarev and David J. Nicholls, "Nonlinearity in Data Related to War," in Supplementary Ways for Increasing International Stability (Vienna, Austria: 29 September - 1 October, 1995), 165-169.

Tsuhan Chen, ed., The Past, Present, and Future of Neural Networks for Signal Processing, IEEE Signal Processing Magazine 14, 9 (November 1997), 28-48.

Todor Tagarev, "Non-linear predictive models as compressors for time-series: A case of classifying qualitative dynamics," in Preprints of the 3rd European IEEE Workshop on Computer-Intensive Methods in Control and Data Processing, ed. J. Rojicek, M. Valeckova, M. Karny and K. Warwick (Prague, September 1998), 29-34.

James B. Kadtke and Michael Kremliovsky, "Classifying Complex, Deterministic Signals," in Predictability of Complex Dynamical Systems, ed. Yurii A. Kravtsov and James B. Kadtke, Springer Series in Synergetics, vol. 69, (Berlin: Springer, 1996).

Petya Ivanova and Todor Tagarev, "Indicator Space Configuration for Early Warning of Violent Political Conflicts by Genetic Algorithms," Annals of Operations Research (to appear).

Tom M. Mitchell, Machine Learning (New York: McGraw-Hill, 1997).

Other issues of using cluster analysis, especially in data mining and knowledge discovery from large data bases, are discussed in detail by David Wishart, "Efficient Hierarchical Cluster Analysis for data Mining and Knowledge Discovery," Computing Science and Statistics 30 (1998), 257-263; David Wishart, "ClustanGraphics3: Interactive Graphics for Cluster Analysis," Classification in the Information Age, W. Gaul and H. Locarek-Junge, ed. (Springer-Verlag, 1999); as well as in other papers in that volume.

31 For detailed discussion see Christopher M. Bishop, Neural Networks for Pattern Recognition (Oxford: Oxford University Press, 1995), 295-331. 
32 For applications to classification and forecasting problems refer to Petya Ivanova, "Feature Selection for Neural Network Forecaster by Genetic Algorithms," Proceedings of the 12th International Conference on Systems Science (Wrozlaw, Poland, 1995), vol. 3, 402-408; Petya Ivanova, Todor Tagarev and Andrew Hunter, "Selection of Indicators for Early Warning of Violent Political Conflicts by Genetic Algorithms," Proceedings of the International Conference on Transition to Advanced Market Institutions and Economies (Warsaw, Poland: June 1997), 180-183.

33 Velizar Shalamanov, "CJTF C4I System for Early Warning and Rapid Reaction," 18th AFCEA Europe Brussels Symposium (Brussels: AFCEA Europe, October 1997).

TODOR TAGAREV is 1982 graduate of the Bulgarian Air Force Academy. In 1989 he received a Ph.D. degree in systems and control from Zhukovsky Air Force Engineering Academy, Moscow. Dr. Tagarev is 1994 Distinguished Graduate of the US Air Command and Staff College and 1994 Distinguished Young AFCEAn. Currently, he is a Senior Researcher at the Department of Nonlinear Dynamics, Space Research Institute of the Bulgarian Academy of Sciences. Dr. Tagarev is Senior Associate of the Institute for Security and International Studies and is involved in a comprehensive study and reengineering of the Bulgarian defense planning system. Specializes in information aspects of security, computer modeling and prediction of complex process, including security and defense related processes. E-mail: infosec@mbox.digsys.bg.

PETYA IVANOVA holds a M.Sc. degree in bioengineering from the Technical University of Sofia, Bulgaria (1992), and a M.Sc. degree in Decision Support Systems from the University of Sunderland, U.K. (1994). She has more than thirty publications, mainly in the application of neural network and evolutionary methods for the purposes of classification and forecasting, and is currently finalizing her Ph.D. dissertation on "Designing Nonlinear Predictors: Chaos Theory and Evolutionary Approaches". Ms Ivanova is currently with the Institute of Information Theory and Automation (UTIA), Academy of Sciences of the Czech Republic, Prague. E-mail: ivanova@utia.cas.cz. 